\title{
THE REPETITIONS APPROACH TO \\ CHARACTERIZE CARDINAL UTILITY
}

\begin{abstract}
Building on previous work of A. Camacho, we give necessary and sufficient conditions for the existence of a cardinal utility function to represent, through summation, a preference relation on sequences of alternatives.
\end{abstract}

\section{INTRODUCTION}

Till recently, there were mainly three ways to derive cardinal utility. One is the approach, using strength of preference as a primitive. A second approach uses lotteries. Thirdly there is the approach where alternatives have several coordinates, and the utility function is a sum of coordinate functions.

Recently Camacho came with a new approach, the repetitions approach. For a careful exposition of this approach, a comparison to other approaches, and an explanation of its intuitive virtues, the reader is referred to Camacho [1-4]. The purpose of this paper is to use the ideas of Camacho to give a set of necessary and sufficient conditions, alternative to his set, and to give some supplement to his work. Where Camacho works with finite sequences, we use infinite sequences with tails $\alpha_{0}$ ("zero"); in Section 3 we shall show that our set-up is in fact equivalent to Camacho's. We only work with these infinite sequences for their convenience in our present mathematical work.

We assume we have a nonempty set $\mathscr{A}$ of alternatives, with one special element $\alpha^{0}$, the "receive nothing" alternative. By $\mathscr{X} \subset \mathscr{A}^{\mathbb{N}}$ we denote the set of those infinite sequences $x=\left(x_{j}\right)_{j \in \mathbb{N}}$, for which

$$
N_{x}:=\sup \left(\{0\} \cup\left\{j \in \mathbb{N}: x_{j} \neq \alpha^{0}\right\}\right)
$$

is finite, so $x$ has a "tail", constant $\alpha^{0}$. Furthermore we assume a binary relation $\geqslant$ on $\mathscr{X}$, called preference relation, present. Usual notations are $x \leqslant y$ for $y \geqslant x, x>y$ for $x \geqslant y \&$ not $y \geqslant x, x \prec y$ for $y \succ x$, and 
$x \approx y$ for $x \geqslant y \& y \geqslant x$. $\geqslant$ is a weak order if it is transitive and complete $(x \geqslant y$ or $y \geqslant x$, for all $x, y \in X)$.

Our purpose is to find a function

$$
u: \mathscr{A} \rightarrow \mathbb{R} \text { s.t. } x \geqslant y \text { iff } \Sigma_{j=1}^{\infty}\left[u\left(x_{j}\right)-u\left(y_{j}\right)\right] \geqslant 0 .
$$

For such a function to exist, $\succcurlyeq$ must certainly satisfy the following four axioms, as can be checked straightforwardly and is not elaborated here.

AXIOM 1. $\succcurlyeq$ is a weak order.

AXIOM 2 (The Permutation Axiom). For all $x, y \in \mathscr{X}, N \in \mathbb{N}$, permutations $\pi$ on $\{1, \ldots, N\}$, s.t. $x_{j}=y_{\pi(j)}$ for all $j \leqslant N, x_{j}=y_{j}$ for all $j>N$; we have $x \approx y$.

(A reordering of alternatives does not change desirability).

AXIOM 3 (The Independence Axiom). For all $x, y, x^{\prime}, y^{\prime} \in \mathscr{X}, i \in \mathbb{N}$, s.t. $x_{i}=y_{i}, \quad x_{i}^{\prime}=y_{i}^{\prime}, \quad x_{j}=x_{j}^{\prime}$ and $y_{j}=y_{j}^{\prime}$ for all $j \neq i$, we have $x \geqslant y \Leftrightarrow x^{\prime} \geqslant y^{\prime}$.

(The preference between $x$ and $y$ is independent of coordinates $i$ at which $x$ and $y$ are equal.)

AXIOM 4 (The Archimedean Axiom). For all $x, y, v, w \in \mathscr{X}$ with $x \succ y$, $v \succ w$, there exists $M \in \mathbb{N}$ s.t. $p \succcurlyeq q$ where $p_{k N_{x}+j}=x_{j}$ for all $0 \leqslant k \leqslant M-1,1 \leqslant j \leqslant N_{x}, p_{M N_{x}+j}=w_{j}$ for all $1 \leqslant j \leqslant N_{w}$, and $p_{n}=\alpha^{0}$ for all $n>M N_{x}+N_{w}$; and where $q_{l N_{y}+i}=y_{i}$ for all $0 \leqslant l \leqslant M-1,1 \leqslant i \leqslant N_{y}, q_{M N_{y}+i}=v_{i}$ for all $1 \leqslant i \leqslant N_{v}$, and $q_{m}=\alpha^{0}$ for all $m>M N_{y}+N_{v}$.

(The difference between $v$ and $w$ can be compensated by a sufficient number of differences between $x$ and $y$.)

Constructions such as that of $p$ above will more often be carried out in the sequel. One can imagine the "untailed" part of $p$ to consist of $M$ replicas of the "untailed" part of $x$, followed by one replica of the "untailed" part of $w$. Axiom 4 has not been used by Camacho, but he indicated it more or less in Section 2.1, page 364, (d), in [3]. 
Our main result:

THEOREM 1.1. The following two assertions are equivalent:

1.1. (i) "There exists $u: \mathscr{A} \rightarrow \mathbb{R}$ s.t.

$$
x \geqslant y \Leftrightarrow \Sigma_{j=1}^{\infty}\left[u\left(x_{j}\right)-u\left(y_{j}\right)\right] \geqslant 0 . "
$$

1.1. (ii) " $\geqslant$ satisfies axioms 1 to 4 ."

Furthermore, if (i) holds, then $u$ can be replaced by $\bar{u}: \mathscr{A} \rightarrow \mathbb{R}$ if and only if real $\tau$ and positive $\sigma$ exist s.t. $\bar{u}=\tau+\sigma u$.

The implication (i) $\Rightarrow$ (ii) is straightforward. In the next section we assume (ii), and derive (i), and the "Furthermore ..." statement.

\section{PROOF OF THEOREM 1.1}

Assume Axioms 1 to 4 are satisfied. We define an equivalence relation $E$ on $\mathscr{X}$ by $x E y$ if $\left\|\left\{j \mid x_{j}=\beta\right\}\right\|=\left\|\left\{j \mid y_{j}=\beta\right\}\right\|$ for all $\beta \neq \alpha^{0}$ in $\mathscr{A}$. By $[x]$ we denote the equivalence class $\{y \in \mathscr{X} \mid y E x\}$, and $[\mathscr{X}]:=\{[x] \mid x \in \mathscr{X}\}$. By the permutation Axiom we have $E \subseteq \approx$. We may write $[x]=$ $\sum_{j=1}^{n} n_{j}\left[\alpha_{j}\right]$, with $n, n_{j} \in \mathbb{N}$ for all $j,\left\{x_{i}: i \in \mathbb{N}\right\}=\left\{\alpha_{j}: 1 \leqslant j \leqslant n\right\} \cup\left\{\alpha^{0}\right\} ;$ and $n_{j}=\left\|\left\{i: x_{i}=\alpha_{j}\right\}\right\|$ if $\alpha_{j} \neq \alpha^{0}$ for all $j$, and $\alpha_{j} \neq \alpha_{k}$ if $j \neq k$. We define $[x]+[y]$, and $n[x]$ for $n \in \mathbb{N} \cup\{0\}$ in the usual way. The operation + on $[\mathscr{X}]$ is associative and commutative, has neutral element $\left[\left(\alpha^{0}, \alpha^{0}, \ldots\right)\right]$.

We define the binary relation $\geqslant^{\prime}$ on $[\mathscr{X}]$ by $[x] \geqslant{ }^{\prime}[y]$ if there exist $v \in[x], w \in[y]$, s.t. $v \geqslant w$. By Axioms 1 and 2 this is iff $v \geqslant w$ for all $v \in[x], w \in[y]$. So we have $x \geqslant y \Leftrightarrow[x] \geqslant{ }^{\prime}[y]$. The notations $\preccurlyeq^{\prime}, \succ^{\prime}$, $\prec^{\prime}, \approx^{\prime}$ are as usual. We have, for all $x, y, v, w \in \mathscr{X}$ :

LEMMA $1^{\prime} . \geqslant^{\prime}$ is a weak order.

LEMMA $2^{\prime} . x \geqslant y \Leftrightarrow[x] \geqslant '[y]$.

LEMMA 3'. (Additivity). $[x] \geqslant^{\prime}[y] \Leftrightarrow[x]+[v] \geqslant[y]+[v]$. 
Proof. $\left(v_{1}, \ldots, v_{N_{v}}, x_{1}, \ldots, x_{N_{x}}, \alpha^{0}, \ldots\right) \in[x]+[v] ;\left(v_{1}, \ldots, v_{N_{v}}\right.$, $\left.y_{1}, \ldots, y_{N_{y}}, \alpha^{0}, \ldots\right) \in[y]+[v] ;\left(\alpha^{0}, \ldots, \alpha^{0}, x_{1}, \ldots, x_{N_{x}}, \alpha^{0}, \ldots\right)$ (first $N_{v}$ coordinates $\left.\alpha^{0}\right) \in[x] ;\left(\alpha^{0}, \ldots, \alpha^{0}, y_{1}, \ldots, y_{N_{y}}, \alpha^{0}, \ldots\right)$ (first $N_{v}$ coordinates $\left.\alpha^{0}\right) \in[y]$. Now apply independence of $\succcurlyeq, N_{v}$ times; then Lemma $2^{\prime}$.

LEMMA 4'. (Archimedean Axiom for $\succcurlyeq^{\prime}$ ). If $[x] \succ^{\prime}[y]$, $[v] \succ^{\prime}[w]$, then $M \in \mathbb{N}$ exists s.t. $M[x]+[w] \succcurlyeq{ }^{\prime} M[y]+[v]$.

Proof. Define $p, q$ as in Axiom 4. Then $p \in M[x]+[w], q \in M[y]+[v]$. Apply Axiom 4, and Lemma 2'.

These four lemmas enable us to apply Theorem 3.2.1.1 of Krantz et al. [5]. In this we do not need commutativity of + .

THEOREM 2.1. For any binary relation $\widetilde{F}^{\prime}$ on $[\mathscr{X}]$ the following two assertions are equivalent.

2.1. (i) “There exists $\phi:[\mathscr{X}] \rightarrow \mathbb{R}$ s.t. $[x] \succcurlyeq '[y] \Leftrightarrow \phi([x]) \geqslant \phi([y])$ and s.t. $\phi([x]+[y])=\phi([x])+\phi([y])$, for all $x, y \in \mathscr{X} . "$

2.1. (ii) " $\succcurlyeq$ ' is a weak order that satisfies additivity and the Archimedean Axiom."

Furthermore, another function $\bar{\phi}$ satisfies (i) if and only if positive $\sigma$ exists s.t. $\bar{\phi}=\sigma \phi$.

Proof. By Theorem 3.2.1.1 of Krantz et al. [5]. Note for this that, if $[w] \geqslant^{\prime}[v]$ and $[x] \succ^{\prime}[y]$, then by repeated application of Lemmas $1^{\prime}$ and $3^{\prime},[x]+[w] \geqslant^{\prime}[y]+[w] \geqslant^{\prime}[y]+[v]$. So still the result of Lemma $4^{\prime}$ holds, with $M=1$.

LEMMA 5. Let $\succcurlyeq$ be a binary relation on $\mathscr{X}, \succcurlyeq$ one on [X] , s.t. $x ₹ y \Leftrightarrow[x] \succcurlyeq[$ [ $y$. Then Assertion 2.1. (i) implies Assertion 1.1. (i) with $₹$ for $\geqslant$, by the definition $u(\alpha):=\phi\left(\left[\alpha, \alpha^{0}, \ldots\right]\right)$. And then Assertion 1.1. (i) with $\succcurlyeq$ for $\geqslant$ implies Assertion 2.1. (i) by the definition

$$
\phi\left(\sum_{j=1}^{n} n_{j}\left[\alpha_{j}\right]\right)=\sum_{j=1}^{n} n_{j}\left[u\left(\alpha_{j}\right)-u\left(\alpha^{0}\right)\right] .
$$

Proof. Let Assertion 2.1. (i) be satisfied. Define $u$ as above. Then 


$$
\begin{aligned}
& x ₹ y \Leftrightarrow[x] \Im^{\prime}[y] \Leftrightarrow\left(N=\max \left\{N_{x}, N_{y}\right\}\right) \Sigma_{j=1}^{N} 1\left[x_{j}\right] \Re^{\prime} \\
& \overbrace{}^{\prime} \sum_{j=1}^{N} 1\left[y_{j}\right] \Leftrightarrow \sum_{j=1}^{N} \phi\left[\left(x_{j}, \alpha^{0}, \ldots\right)\right] \geqslant \sum_{j=1}^{N} \phi\left[\left(y_{j}, \alpha^{0}, \ldots\right)\right] \Leftrightarrow \\
& \Leftrightarrow \sum_{j=1}^{\infty}\left[u\left(x_{j}\right)-u\left(y_{j}\right)\right] \geqslant 0 \text { : Assertion 1.1. (i). }
\end{aligned}
$$

Next let Assertion 1.1. (i) be satisfied, with $\succcurlyeq$ for $\succcurlyeq$. Define $\phi$ as above. Then $\phi([x]+[y])=\phi([x])+\phi([y])$ for all $x, y$. And

$$
\begin{aligned}
& \phi\left(\sum_{j=1}^{n} n_{j}\left[\alpha_{j}\right]\right) \geqslant \phi\left(\sum_{i=1}^{m} m_{i}\left[\beta_{i}\right]\right) \Leftrightarrow \sum_{j=1}^{n} n_{j}\left[u\left(\alpha_{j}\right)-u\left(\alpha^{0}\right)\right] \geqslant \\
& \geqslant \sum_{i=1}^{m} m_{i}\left[u\left(\beta_{i}\right)-u\left(\alpha^{0}\right)\right] \Leftrightarrow\left\{\text { let } x_{\left(\Sigma_{k=1}^{i-1} n_{k}\right)+n_{j}^{\prime}}=\alpha_{j}\right. \text { for all } \\
& 1 \leqslant j \leqslant n, 1 \leqslant n_{j}^{\prime} \leqslant n_{j}, x_{a}=\alpha^{0} \text { for all } a>\Sigma_{k=1}^{n} n_{k} \text {; } \\
& \left.y \text { analogously from } m, i, \beta_{i} \text { i.s.o. } n, j, \alpha_{j}\right\} \Sigma_{j=1}^{\infty}\left[u\left(x_{j}\right)-\right. \\
& \left.-u\left(y_{j}\right)\right] \geqslant 0 \Leftrightarrow x \succcurlyeq y \Leftrightarrow[x] \succcurlyeq^{\prime}[y] \Leftrightarrow \Sigma_{j=1}^{n} n_{j}\left[\alpha_{j}\right] \succcurlyeq^{\prime} \\
& \mho^{\prime} \sum_{i=1}^{m} m_{i}\left[\beta_{i}\right] \text { : Assertion 2.1. (i). }
\end{aligned}
$$

Now we can complete the proof of Theorem 1.1. Assertion (ii) there implies Assertion 2.1. (ii), as we saw by Lemmas 1' to $4^{\prime}$. Thus it implies Assertion 2.1. (i). Lemma 5 now gives Assertion 1.1. (i). That function $u$, satisfying 1.1. (i), can be replaced by any $\bar{u}=\tau+\sigma u$ for real $\tau$ and positive $\sigma$, is straightforward. Conversely, suppose $u$ in 1.1. (i) can be replaced by $\tilde{u}$. Then derive $\phi$ from $u$ as in Lemma 5 , and analogously $\bar{\phi}$ from $\bar{u}$. By Theorem 2.1 we get that $\bar{\phi}=\sigma \phi$ for a positive real $\sigma$. This can only be if $\bar{u}=\tau+\sigma u$ with $\tau=\bar{u}\left(\alpha^{0}\right)-\sigma u\left(\alpha^{0}\right)$.

\section{EQUIVALENCE OF OUR SET-UP WITH CAMACHO'S}

First we formulate some consequences of Axioms 1 to 4, which directly follow from Theorem 1.1.

DEFINITION. We say $\geqslant$ satisfies the Repetitions Axiom if $[x \geqslant y \Leftrightarrow$ $\left.x^{\prime} \geqslant y^{\prime}\right]$ for all $x, y, x^{\prime}, y^{\prime} \in \mathscr{X}$ for which $n, m \in \mathbb{N}$ exist s.t. $x \geqslant y$, $m \geqslant N_{x}, \quad m \geqslant N_{y}, \quad x_{k m+j}^{\prime}=x_{j}$ and $y_{k m+j}^{\prime}=y_{j}$ for all $1 \leqslant j \leqslant m$, $0 \leqslant k \leqslant n-1, x_{i}^{\prime}=y_{i}^{\prime}=\alpha^{0}$ for all $i>n m$.

COROLLARY 1. Axioms 1 to 4 imply the Repetitions Axiom.

Proof. $\quad x \geqslant y \Leftrightarrow \Sigma_{j=1}^{\infty}\left[u\left(x_{j}\right)-u\left(y_{j}\right)\right] \geqslant 0 \Leftrightarrow n \Sigma_{j=1}^{\infty}\left[u\left(x_{j}\right)-\right.$

$$
\left.u\left(y_{j}\right)\right] \geqslant 0 \Leftrightarrow \sum_{j=1}^{\infty}\left[u\left(x_{j}^{\prime}\right)-u\left(y_{j}^{\prime}\right)\right] \geqslant 0 \Leftrightarrow x^{\prime} \geqslant y^{\prime} \text {. }
$$


DEFINITION. We say $\geqslant$ satisfies the Rate of Substitution Axiom if for all $\alpha, \beta, \gamma, \delta \in \mathscr{A}$ with $\left(\alpha, \alpha^{0}, \ldots\right) \geqslant\left(\beta, \alpha^{0}, \ldots\right)$ and $\left(\gamma, \alpha^{0}, \ldots\right) \succ$ $\left(\delta, \alpha^{0}, \ldots\right)$, there exists $R(\alpha, \beta, \gamma, \delta) \in \mathbb{R}$ s.t. for all $x, y \in \mathscr{X}, n$, $m \in \mathbb{N} \cup\{0\}, A, B \subset \mathbb{N}$ with $A \cap B=\phi,\|A\|=n>0,\|B\|=m$, $x_{j}=y_{j}$ for all $j \notin A \cup B, x_{j}=\alpha$ and $y_{j}=\beta$ for all $j \in A, x_{j}=\delta$ and $y_{j}=\gamma$ for all $j \in B$, we have $x>y$ iff $m / n<R(\alpha, \beta, \gamma, \delta), x \approx y$ iff $m / n=R(\alpha, \beta, \gamma, \delta)$, and $x<y$ iff $m / n>R(\alpha, \beta, \gamma, \delta)$.

COROLLARY 2. Axioms 1 to 4 imply the Rate of Substitution Axiom.

Proof. Let, for $\alpha, \beta, \gamma, \delta$ as above, $R(\alpha, \beta, \gamma, \delta)=[u(\alpha)-u(\beta)] /$ $[u(\gamma)-u(\delta)]$.

In Camacho [2-4], also a nonempty set $\mathscr{A}$ is the point of departure, but the set $\mathscr{X}^{f}:=\bigcup_{n=1}^{\infty} \mathscr{A}^{n}$ of all finite sequences of alternatives is considered. If $x \in \mathscr{A}^{n}$, we say $x$ has length $n$. There is assumed to be a binary (preference) relation $\succcurlyeq$ " present on $\mathscr{X}^{f}$, such that the restriction of $\succcurlyeq$ " to $\mathscr{A}^{n}$ is a weak order for every $n \in \mathbb{N}$, and such that $x \geqslant " y$ for no $x, y$ of different length. From Camacho's set-up we can come to our approach as follows. Take an arbitrary element of $\mathscr{A}$, denote it as $\alpha^{0}$. Assign to every $\left(x_{1}, \ldots, x_{n}\right) \in \mathscr{X}^{f}$ the element $\left(x_{1}, \ldots, x_{n}, \alpha^{0}, \ldots\right)$ of $\mathscr{X}$. And write $x \geqslant y$ if $\left(x_{1}, \ldots, x_{n}\right) \geqslant "\left(y_{1}, \ldots, y_{n}\right)$, with $n=\max \left\{N_{x}, N_{y}\right\}$, for all $x, y \in \mathscr{X}$.

Conversely, from our set-up we can come to Camacho's set-up by defining $x \geqslant " y$ whenever

$$
\left(x_{1}, \ldots, x_{n}, \alpha^{0}, \ldots\right) \geqslant\left(y_{1}, \ldots, y_{n}, \alpha^{0}, \ldots\right) \text {, }
$$

for all $x, y \in \mathscr{A}^{n}$; for all $n \in \mathbb{N}$.

Now the combination of our weak order and independence Axioms for $\geqslant$ is equivalent to the combination of Camacho's weak order and independence Axioms for $\succcurlyeq "$. For brevity we do not elaborate that here. Also, in the presence of weak orderness and independence, the permutation, repetition, and rate of substitution Axioms in our set-up are equivalent to those in Camacho's set-up. The key role in all this is played by the independence Axioms. 


\section{AN EXAMPLE}

In this section we give an example to illustrate the necessity of the Archimedean Axiom. Also this should illustrate where we deviate from Camacho's set-up. Let $\mathscr{A}=\left\{\alpha^{0}, \beta, \gamma, \delta, \varepsilon\right\}$, and let $f: \mathscr{A} \rightarrow \mathbb{R}$ be s.t. $f\left(\alpha^{0}\right)=0, f(\beta)=1, f(\gamma)=\sqrt{2}, f(\delta)=\sqrt{3}, f(\varepsilon)=1+\sqrt{2}+\sqrt{3}$. For $x, y \in \mathscr{X}$, we have $x>y$ if $\Sigma_{j=1}^{\infty}\left[f\left(x_{j}\right)-f\left(y_{j}\right)\right]>0$ or $\sum_{j=1}^{\infty}\left[f\left(x_{j}\right)-\right.$ $\left.f\left(y_{j}\right)\right]=0$ and $\left\|\left\{j: x_{j}=\varepsilon\right\}\right\|>\left\|\left\{j: y_{j}=\varepsilon\right\}\right\|$. We have $x \approx y$ if $\sum_{j=1}^{\infty}\left[f\left(x_{j}\right)-f\left(y_{j}\right)\right]=0$ and $\left\|\left\{j: x_{j}=\varepsilon\right\}\right\|=\left\|\left\{j: y_{j}=\varepsilon\right\}\right\|$, which can be seen to occur only if $x E y$, with $E$ as in Section 2. Of course $x \geqslant y$ if $x>y$ or $x \approx y$. It can be seen that $\geqslant$ is a weak order, it satisfies the permutation and independence Axioms. But it does not satisfy the Archimedean Axiom. To see this, take $x=\left(\varepsilon, \alpha^{0}, \ldots\right), y=\left(\beta, \gamma, \delta, \alpha^{0}, \ldots\right)$, $v=\left(\beta, \alpha^{0}, \ldots\right), w=\left(\alpha^{0}, \alpha^{0}, \ldots\right)$. Then $x>y, v>w$, but for all $M \in \mathbb{N}$, and $p, q$ as in Axiom 4 we have $p \prec q$ since

$$
\Sigma_{j=1}^{\infty}\left[f\left(p_{j}\right)-f\left(q_{j}\right)\right]=M \Sigma_{j=1}^{\infty}\left[f\left(x_{j}\right)-f\left(y_{j}\right)\right]+f(w)-f(v)<0 .
$$

So Axiom 4 is violated. It can easily be seen that $\geqslant$ satisfies the Repetitions Axiom, this in fact is implied by Axioms 1 to 3 . We finally show that $\succcurlyeq$ satisfies the Rate of Substitution Axiom. To every four $\mu, v, \sigma, \tau$ in $\mathscr{A}$ s.t. $\left(\mu, \alpha^{0}, \ldots\right) \geqslant\left(\nu, \alpha^{0}, \ldots\right)$ and $\left(\sigma, \alpha^{0}, \ldots\right) \succ\left(\tau, \alpha^{0}, \ldots\right)$, we assign

$$
R(\mu, v, \sigma, \tau):=[f(\mu)-f(v)] /[f(\sigma)-f(\tau)] .
$$

Let then $x, y \in \mathscr{X}, n, m \in \mathbb{N} \cup\{0\}, A, B \subset \mathbb{N}$ with $\|A\|=n>0$, $\|B\|=m, x_{j}=y_{j}$ for all $j \notin A \cup B, x_{j}=\mu$ and $y_{j}=v$ for all $j \in A$, $x_{j}=\tau$ and $y_{j}=\sigma$ for all $j \in B$. If now $m / n<R(\mu, v, \sigma, \tau)$, then

$$
\sum_{j=1}^{\infty}\left[f\left(x_{j}\right)-f\left(y_{j}\right)\right]=n[f(\mu)-f(v)]-m[f(\sigma)-f(\tau)]>0,
$$

so $x>y$. If $m / n>R(\mu, v, \sigma, \tau)$, then analogously $x<y$.

Remains the case $m / n=R(\mu, v, \sigma, \tau)$.

Apparently then $[f(\mu)-f(v)] /[f(\sigma)-f(\tau)]$ is rational. There are only a few possibilities for this: either $\mu=v$, or $\mu=\sigma$ and $v=\tau$. In either case $x \approx y$ follows.

\section{REFERENCES}

[1] Camacho, A.: 1979, 'Maximizing Expected Utility and the Rule of Long Run Success', in Maurice Allais and Ole Hagen (eds.), Expected Utility and the Allais Paradox, D. Reidel, Dordrecht. 
[2] Camacho, A.: 1979, 'On Cardinal Utility', Theory and Decision 10, 131-145.

[3] Camacho, A.: 1980, 'Approaches to Cardinal Utility', Theory and Decision 12, 359-379.

[4] Camacho, A., 1982, Societies and Social Decision Functions, D. Reidel, Dordrecht.

[5] Krantz, D. H., Luce, R. D., Suppes; P., and Tversky, A.: 1971, Foundations of Measurement, Vol. I, Academic Press, New York.

Department of Mathematics,

University of Nijmegen,

Toernooiveld,

6525 ED Nijmegen,

The Netherlands 